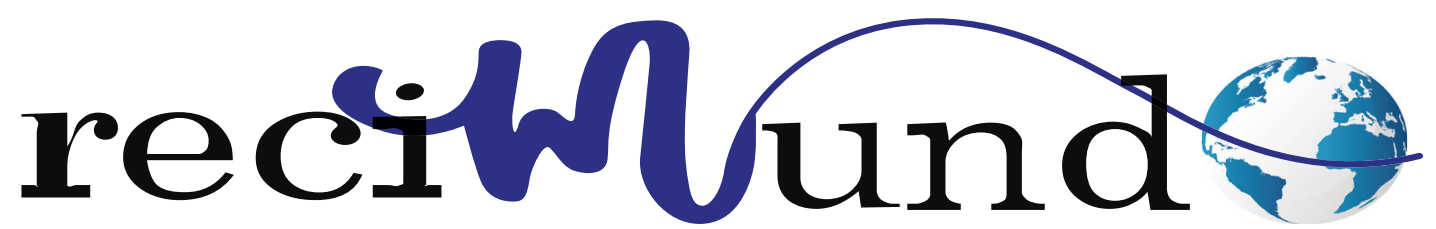

Revista Científica Mundo de la Investigación y el Conocimiento

DOl: 10.26820/recimundo/5.(2).abril.2021.80-88

URL: https://recimundo.com/index.php/es/article/view/1043

EDITORIAL: Saberes del Conocimiento

REVISTA: RECIMUNDO

ISSN: 2588-073X

TIPO DE INVESTIGACIÓN: Artículo de revisión

CóDIGO UNESCO: 32 Ciencias Médicas

PAGINAS: $80-88$

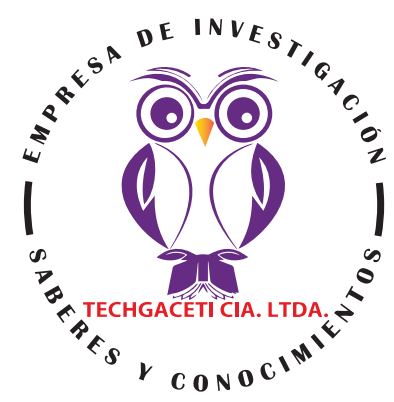

\title{
Manejo farmacológico en epilepsia de origen tumoral
}

Pharmacological management in epilepsy of tumor origin

Manejo farmacológico em epilepsia de origem tumoral

Andrés Fernando Morales Sánchez1; Jorge Luis Morales Sánchez²; Stalin Gabriel Guerra Hurtado3;

Karla Abigail Navarrete Bolaños ${ }^{4}$

\section{RECIBIDO: 15/01/2021 ACEPTADO: 20/03/2021 PUBLICADO: 01/04/2021}

1. Médico General en Libre Ejercicio; Pelileo, Ecuador; morales.saf94@outlook.com; iD https://orcid.org/0000-0003-09631776

2. Médico Ocupacional (E) y Médico General en Atención Primaria Salud Distrito Salud 18D04; Pelileo; Ecuador; mdjorgemoraless@hotmail.com; (iD) https://orcid.org/0000-0003-1846-7744

3. Médico Rural, puesto de salud Yaapi; Quito, Ecuador; stalinguerra17@gmail.com; (D) https://orcid.org/0000-0003-42162211

4. Médico General en Libre Ejercicio; Ambato, Ecuador; karlita_navarreteb@hotmail.com; (ID https://orcid.org/0000-00024365-160X

\section{CORRESPONDENCIA}

Andrés Fernando Morales Sánchez

morales.saf94@outlook.com

Pelileo, Ecuador

(c) RECIMUNDO; Editorial Saberes del Conocimiento, 2021 


\section{RESUMEN}

La epilepsia se define como una afección cerebral crónica caracterizada por crisis recurrentes de etiología heterogénea (OMS) y afecta a más de 50 millones de personas en el mundo. El 80\% de las personas que la padecen viven en países en vías de desarrollo. En Latinoamérica hay cerca de 5 millones de pacientes con epilepsia, de los cuales hasta el $90 \%$ podría no recibir el tratamiento adecuado. La epilepsia es un trastorno neurológico común en el cual se caracteriza por crisis recurrentes debido a una actividad eléctrica anormal en el cerebro, su etiología es diversa que se acompaña de convulsiones con movimientos bruscos hasta la pérdida de conocimiento. La metodología de la investigación, es una revisión bibliográfica, que se apoyó en medios electrónicos para la búsqueda de la información relevante sobre el objeto de estudio. Entre las conclusiones se tienen que hay muchos medicamentos disponibles para tratar la epilepsia, sin embargo, su uso dependerá del grado de la misma y otras patologías que pueda presentar el paciente. El tratamiento para pacientes en edad avanzada, es más complejo, ya que la persona a mayor edad va perdiendo ciertas condiciones neurológicas y fisiológicas, como el deterioro de la función renal, deterioro del grado cognitivo, entre otros. La incidencia de tumores cerebrales en personas con epilepsia es del 4\%. La epilepsia es la primera manifestación clínica de los tumores cerebrales, entre el 30 y el $50 \%$ de los casos. Para estos casos se puede utilizar la farmacoterapia y el tratamiento quirúrgico el cual su efectividad esta entre un 40 y 90\% de liberación de crisis a los pacientes, pero esta última es más costosa.

Palabras clave: Epilepsia, Neurológico, Trastorno, Convulsión, Cerebro.

\section{ABSTRACT}

Epilepsy is defined as a chronic brain condition characterized by recurrent seizures of heterogeneous etiology (WHO) and affects more than 50 million people in the world. 80\% of the people who suffer from it live in developing countries. In Latin America there are about 5 million patients with epilepsy, of which up to $90 \%$ may not receive adequate treatment. Epilepsy is a common neurological disorder in which it is characterized by recurrent seizures due to abnormal electrical activity in the brain, its etiology is diverse and is accompanied by seizures with sudden movements up to loss of consciousness. The research methodology is a bibliographic review, which relied on electronic means to search for relevant information about the object of study. Among the conclusions are that there are many medications available to treat epilepsy, however, their use will depend on the degree of it and other pathologies that the patient may present. Treatment for elderly patients is more complex, since the older person loses certain neurological and physiological conditions, such as impaired kidney function, cognitive impairment, among others. The incidence of brain tumors in people with epilepsy is 4\%. Epilepsy is the first clinical manifestation of brain tumors, between 30 and $50 \%$ of cases. For these cases, drug therapy and surgical treatment can be used, whose effectiveness is between 40 and $90 \%$ release of seizures to patients, but the latter is more expensive.

Keywords: Epilepsy, Neurological, Disorder, Seizure, Brain.

\section{RESUMO}

A epilepsia é definida como uma condição cerebral crônica caracterizada por crises recorrentes de etiologia heterogênea (OMS) e afeta mais de 50 milhões de pessoas no mundo. 80\% das pessoas que sofrem com ela vivem em países em desenvolvimento. Na América Latina há cerca de 5 milhões de pacientes com epilepsia, dos quais até 90\% podem não receber tratamento adequado. A epilepsia é um distúrbio neurológico comum no qual é caracterizada por crises recorrentes devido a atividade elétrica anormal no cérebro, sua etiologia é diversa e é acompanhada por crises com movimentos bruscos até a perda da consciência. A metodologia de pesquisa é uma revisão bibliográfica, que se baseou em meios eletrônicos para buscar informações relevantes sobre o objeto de estudo. Entre as conclusões estão que existem muitos medicamentos disponíveis para tratar a epilepsia, no entanto, seu uso dependerá do grau de epilepsia e de outras patologias que o paciente possa apresentar. O tratamento de pacientes idosos é mais complexo, já que a pessoa idosa perde certas condições neurológicas e fisiológicas, tais como a deficiência da função renal, deficiência cognitiva, entre outras. A incidência de tumores cerebrais em pessoas com epilepsia é de $4 \%$. A epilepsia é a primeira manifestação clínica de tumores cerebrais, entre 30 e $50 \%$ dos casos. Para estes casos, pode-se utilizar a terapia medicamentosa e o tratamento cirúrgico, cuja eficácia está entre 40 e 90\% de liberação de convulsões para os pacientes, mas esta última é mais cara.

Palavras-chave: Epilepsia, Neurológica, Desordem, Ataque, Cérebro. 


\section{Introducción}

La epilepsia es un trastorno neurológico común en el cual se caracteriza por crisis recurrentes debido a una actividad eléctrica anormal en el cerebro, su etiología es diversa que se acompaña de convulsiones con movimientos bruscos hasta la pérdida de conocimiento (LAPO ASENCIO, 2019). La epilepsia se define como una afección cerebral crónica caracterizada por crisis recurrentes de etiología heterogénea (OMS) y afecta a más de 50 millones de personas en el mundo. El $80 \%$ de las personas que la padecen viven en países en vías de desarrollo. En Latinoamérica hay cerca de 5 millones de pacientes con epilepsia, de los cuales hasta el 90\% podría no recibir el tratamiento adecuado (Lozano Tangua, 2013).
De acuerdo con la última clasificación de la Liga Internacional contra la Epilepsia (2017), se puede clasificar la epilepsia en los tipos focal, generalizada o de origen desconocido según sea patrón clínico de inicio de las crisis y los hallazgos electrodiagnósticos complementarios. La epilepsia del lóbulo temporal es el tipo de epilepsia focal de más alta incidencia demostrada en estudios epidemiológicos en centros especializados en el manejo de esta patología. En unidades de cirugía de epilepsia, la epilepsia del lóbulo temporal puede llegar a representar aproximadamente un $66 \%$ de los casos de epilepsias farmacorresistentes que requieren del manejo quirúrgico (el grueso de esta casuística corresponde al caso de la epilepsia temporal mesial) (Víquez \& Segura, 2017).

Tabla 1. Características de la epilepsia temporal mesial y lateral.

\begin{tabular}{|c|c|c|}
\hline & Epilepsia temporal mesial & Epilepsia temporal lateral \\
\hline $\begin{array}{c}\text { Características } \\
\text { clínicas }\end{array}$ & $\begin{array}{l}\text { Historia en la infancia de crisis febriles } \\
\text { (relacionada a esclerosis hipocampal). } \\
\text { Auras comunes: viscerales, cefálicas, } \\
\text { gustativas, afectivas, olfatorias, fenómenos } \\
\text { de déjà vu y jamais vu. } \\
\text { Preservación parcial de la consciencia en } \\
\text { fases tempranas de crisis. } \\
\text { Evolución lenta de la crisis. Arresto motor } \\
\text { prominente. Disfasia y confusión postictal } \\
\text { prominentes. } \\
\text { Posición distónica del miembro superior } \\
\text { contralateral. } \\
\text { Síntomas autonómicos (palidez, rubor, } \\
\text { taquicardia). } \\
\text { Automatismos oroalimentarios } \\
\text { gesticulares. }\end{array}$ & $\begin{array}{l}\text { Típicamente sin historia de } \\
\text { crisis febriles en infancia. } \\
\text { Auras comunes: alucinaciones o } \\
\text { ilusiones, (auditivas, } \\
\text { percepciones complejas o } \\
\text { experimentales). } \\
\text { Arresto motor y automatismos } \\
\text { similares a epilepsia temporal } \\
\text { mesial. }\end{array}$ \\
\hline
\end{tabular}




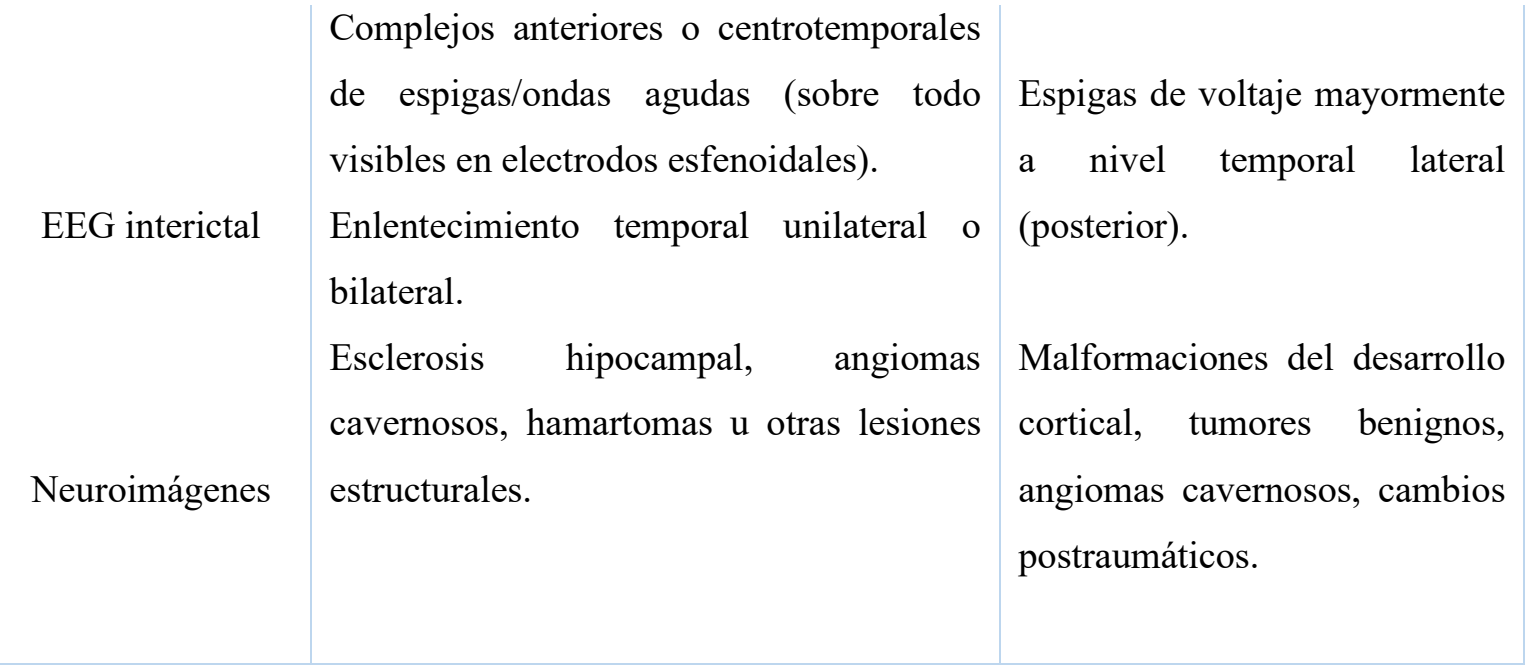

Fuente: (Víquez \& Segura, 2017)

El 20 - 30\% de los pacientes no se controlan de manera adecuada con el tratamiento farmacológico, convirtiéndose en candidatos para cirugía de epilepsia. En ELT (epilepsia del lóbulo temporal) el manejo quirúrgico es más costo-efectivo que el tratamiento médico continuo, consiguiendo entre un $40 \%$ y 90\% de pacientes libres de crisis (Lozano Tangua, 2013).

La incidencia de tumores cerebrales en personas con epilepsia es del $4 \%$, y en los enfermos con neoplasias intracraneanas la frecuencia de estas crisis es del $30 \%$ o más, dependiendo del tipo de tumor. La epilepsia es la primera manifestación clínica de los tumores cerebrales, entre el 30 y el $50 \%$ de los casos; en un 10 y $30 \%$ de enfermos las convulsiones se expresan tardíamente en el curso de la enfermedad (Herrera, y otros, s.f).

En pacientes con ELT operados, la esclerosis del hipocampo se asocia a la presencia de una lesión cerebral temprana (LCT) en la que en un 53\% de los casos está involucrada una convulsión. En los pacientes con ELT idiopática, sin antecedente de LCT, se observa una menor pérdida neuronal y un peor pronóstico posquirúrgico. Esto sugiere que la fisiopatología de la esclerosis del hipocampo se relaciona con la presencia de una LCT debida a una enfermedad cerebral o médica en las etapas iniciales del desarrollo. Tras el daño hipocámpico inicial se produce una pérdida neuronal subsiguiente. Sin embargo, aún no es posible determinar qué parte de la lesión hipocámpica encontrada en los pacientes tras la cirugía se debe a la LCT y qué parte corresponde a los cambios isquémicos secundarios (Volcy-Gómez, 2014).

Una de las causas de crisis epilépticas son las neoplasias. Las manifestaciones clínicas se correlacionan al sitio donde se encuentra la lesión neoplásica, según descriptores clínicos motores, sensitivos y de automatismos, entre otros, con o sin generalización secundaria. Es frecuente que este tipo de epilepsia sea refractaria, siendo necesario el uso de más de dos antiepilépticos a dosis altas (Peña, 2018).

Se ha establecido, además, el término de "tumores asociados a epilepsia crónica", con el que se describen pacientes con historia de epilepsia de larga evolución, de aparecimiento en edades jóvenes, de carácter usualmente focal, de tipo refractario, con identificación de lesiones neoplásicos por neuroimagen (Peña, 2018). 


\section{Metodología}

La metodología de la investigación, es una revisión de tipo bibliográfica, que ha sido apoyada en medios electrónicos, para la obtención primaria de la información, lo que se busca es hacer una síntesis de aquellos trabajos de investigación que abordan el tema de estudio, y que se consideren hagan aportes importantes para el presente trabajo, y que sirva de punto de partida para futuras investigaciones.

\section{Resultados}

\section{Manejo farmacológico}

En las últimas dos décadas ha habido avances importantes en el campo de la investigación que han culminado en un mejor entendimiento y tratamiento del estatus epiléptico. Uno de los grandes logros está relacionado con los nuevos intentos en unificar la definición basada en la duración de la crisis, lo que ha permitido intervenciones farmacológicas más eficaces en los campos pre-hospitalario y hospitalario (servicios de urgencias y unidades de cuidado intensivo) (González \& Rodríguez, 2011).

\section{Manejo pre hospitalario}

- El manejo básico esta dado por valoración de la vía aérea, la respiración y la circulación (ABC).

- Succión de las secreciones, la administración de oxígeno suplementario, acceso intravenoso e inicio de antiepilépticos.

- Indagar situaciones de alto riesgo tales como historia de enfermedad cardiaca, historia de crisis epilépticas previas, embarazo, diabetes, trauma o sobredosis de cualquier fármaco o tóxico.

- Durante el transporte el paciente debe recibir soporte de los signos vitales, descartando además situaciones de riesgo como hipoglicemia o hipoxemia.

- Aquellos pacientes que tienen historia de diabetes no deben recibir glucosa empíricamente y se deberá esperar confirmación de los análisis de glucometría (González \& Rodríguez, 2011).

\section{Manejo en el Servicio de Urgencias}

- Al ingresar al servicio de urgencias se debe administrar una benzodiacepina, si ésta ya ha sido administrada por el personal paramédico se puede repetir la dosis.

- Además, los pacientes deben recibir $100 \mathrm{mg}$ de tiamina y $50 \mathrm{ml}$ de dextrosa al $50 \%$ si la glucosa es baja o indeterminada especialmente ante la sospecha de alcoholismo.

- Se deben hacer pruebas para determinar niveles de electrolitos (sodio, calcio, magnesio, potasio, fósforo), función miocárdica, CPK total, gases arteriales, niveles de amonio, transaminasas, creatinina, ácido láctico, hemograma total y exámenes para descartar tóxicos.

- En aquellos pacientes con antecedentes de epilepsia deben obtenerse los niveles de antiepilépticos (González \& Rodríguez, 2011). 
Tabla 2. Dosis de inicio y mantenimiento de medicaciones comúnmente utilizadas en estatus epiléptico.

\begin{tabular}{|c|c|c|c|}
\hline Medicación & Dosis de carga & Infusión & $\begin{array}{l}\text { Efecto secundario } \\
\text { importante }\end{array}$ \\
\hline Lorazepam & $0.1 \mathrm{mg} / \mathrm{kg}$ & $2 \mathrm{mg} /$ minuto & $\begin{array}{l}\text { Sedación } \\
\text { Depresión respiratoria }\end{array}$ \\
\hline Diazepam & $0.2 \mathrm{mg} / \mathrm{kg}$ & $5 \mathrm{mg} /$ minuto & $\begin{array}{l}\text { Sedación } \\
\text { Depresión respiratoria }\end{array}$ \\
\hline Clonazepam & $1-2 \mathrm{mg}$ & $\begin{array}{c}0.05-0.1 \\
\mathrm{mg} / / \mathrm{k} / \mathrm{hora}\end{array}$ & $\begin{array}{l}\text { Sedación } \\
\text { Depresión respiratoria }\end{array}$ \\
\hline Fosfenitoina & $20 \mathrm{mg} / \mathrm{kg}$ & $150 \mathrm{mg} /$ minuto & $\begin{array}{l}\text { Hipotensión } \\
\text { Arritmia cardiaca }\end{array}$ \\
\hline Fenitoina & $20 \mathrm{mg} / \mathrm{kg}$ & $50 \mathrm{mg} /$ minuto & $\begin{array}{l}\text { Hipotensión } \\
\text { Arritmia cardiaca } \\
\text { Irritación local }\end{array}$ \\
\hline Acido Valproico & $25-45 \mathrm{mg} / \mathrm{Kg}$ & $\begin{array}{c}\text { Hasta } 6 \\
\mathrm{mg} / \mathrm{k} / \mathrm{minuto}\end{array}$ & $\begin{array}{l}\text { Encefalopatía severa } \\
\text { (pacientes con hiperamonemia } \\
\text { o enfermedad mitocondrial) }\end{array}$ \\
\hline Levetiracetam & $\begin{array}{l}20 \mathrm{mg} / \mathrm{kg} \text {, } \\
\text { (usualmente } 2 \mathrm{gr} \text { ) }\end{array}$ & $\begin{array}{l}\text { Entre 5-15 } \\
\text { minutos }\end{array}$ & Sedación leve \\
\hline Lacosamida & $200-400 \mathrm{mg}$ & $\begin{array}{l}\text { Entre } 40-80 \\
\mathrm{mg} / \mathrm{min}(10-30 \\
\text { minutos })\end{array}$ & Sin efectos serios reportados \\
\hline Midazolam & $0.2 \mathrm{mg} / \mathrm{kg}$ & $0.2-5 \mathrm{mg} / \mathrm{k}$ & $\begin{array}{l}\text { Sedación } \\
\text { Hipotensión } \\
\text { Depresión respiratoria }\end{array}$ \\
\hline Propofol & $2 \mathrm{mg} / \mathrm{kg}$ & $2-10 \mathrm{mg} / \mathrm{k} / \mathrm{hora}$ & $\begin{array}{l}\text { Sedación } \\
\text { Hipotensión } \\
\text { Depresión respiratoria } \\
\text { Síndrome de infusión }\end{array}$ \\
\hline Pentobarbital & $5-10 \mathrm{mg} / \mathrm{kg}$ & $1-5 \mathrm{mg} / \mathrm{k} / \mathrm{hora}$ & $\begin{array}{l}\text { Sedación prolongada } \\
\text { Hipotensión } \\
\text { Depresión respiratoria y } \\
\text { miocárdica } \\
\text { Infecciones } \\
\text { Falla hepática } \\
\text { Ileo } \\
\text { Interacción farmacológica }\end{array}$ \\
\hline
\end{tabular}

Fuente: (González \& Rodríguez, 2011) 
El tratamiento de las epilepsias se puede resumir en tres grandes grupos: Profiláctico/preventivo, farmacológico, no farmacológico: cirugía y tratamientos alternativos, sin dejar de mencionarse, el manejo psicológico/psiquiátrico.

\section{Farmacoterapia}

Se debe tener presente en este tema que la terapia en esta enfermedad es todavía supresora, sintomática y no curativa. Se detallan en cada período, la introducción de los diferentes fármacos/procederes en la práctica clínica.

- 1850 bromuros/Hidrato de cloral/Borax, 1910 Fenobarbital, 1930 Dieta Cetogénica, 1938 Fenitoína, 1941 Acetazolamida, 1944 Trimetadiona, 1950 Hormona adrenocorticotropa (ACTH), 1954 Primidona, 1957 Metosuximida, 1958 Ethosuximida, 1962 Sultiamo, 1963 Diazepán, 1965 Carbamazepina, 1967 Ácido Valproico, 1968 Clonazepán, 1975 Clobazán (Bender del Busto \& Hernández Toledo, 2017).

\section{Drogas introducidas entre 1989 y 1994}

- Vigabatrina (1989), Lamotrigina (1990), Oxcarbazepina (1990), Felbamato (1993) y Gabapentina (1994).25 Drogas introducidas entre 1995 y 2008

- Topiramato (1995), Tiagabina (1996), Levetiracetam (1999) Zonisamida (2000), Pregabalina (2004), Stiripentol (2007), Rufinamida (2007), Lacosamida (2008).

Otros fármacos antiepilépticos que se encuentran actualmente en desarrollo activo: Retigabina, Eslicarbazepina, Fluorofelbamato, Remacemida, Valprocemida, Propilsopropil Acetamida. Brivaracetam, Seletracetam, Carisbamato, Ezogabina, Ganaloxona, TPerampanel, T-2000, 2-deoxy-D-glucose, Huperzine A, ICSC 700-008, NAX-5055, NS1209, Tonabersat e YKP3089 (Bender del Busto \& Hernández Toledo, 2017).

\section{En fase experimental se encuentran}

- CPP-115 (derivado Vigabatrina) ácido (1S,3S)-3-amino-4-difluoromethylenylcyclopentanoico

- AGENTES ANTINFLAMATORIOS: HE3286 (Triolex) androsteno-3 $\beta, 7 \beta, 17 \beta$ triol $(\beta \mathrm{AET})$ - VX-765 ácido pirrolidino-2-carboxilico

- otros nueVOS COMPONENTES: 2-Deoxy-D-Glucose, Everolimus, NAX 810-2, Hemisuccinato Propofol, Tonabersat, Isovaleramida, Losigamona, Safinamida, Talampanel (Bender del Busto \& Hernández Toledo, 2017).

\section{Aspectos generales del tratamiento}

- Restricciones en la conducción de vehículos y otras actividades inseguras, la persistencia del estigma y la posibilidad pequeña pero real de la muerte súbita.

- Efectos crónicos indeseados de las DAE sobre la capacidad cognitiva, estado de ánimo, el peso (ganancia y pérdida), la maternidad y la función sexual.

- Estrés cultural y financiero, y algunos regímenes de DAE pueden ser inconvenientes y ponen en peligro el cumplimiento (Bender del Busto \& Hernández Toledo, 2017).

\section{Errores en el tratamiento antiepiléptico}

- Diagnóstico positivo inadecuado.

- No iniciarse al realizar el diagnóstico (esperar el EEG o estudios imagenológicos para imponerlo). Instaurar medicación a dosis total de entrada y no progresiva.

- Uso inicial de politerapia.

- No elección de la droga según tipo de ataque, síndrome o grupo especial.

- Tratamiento de una crisis única sin análisis individualizado.

- Interrumpirle: durante la pubertad, sin período libre de crisis o súbitamente (sustitución brusca).

- No tener en cuenta la vida media de los antiepilépticos y sus niveles plasmáticos. 
- No tener en cuenta la interacción de fármacos, ni la combinación de medicamentos con efectos similares.

- Uso de medicamentos que disminuyan el umbral epileptógeno.

- No tener en cuenta los efectos colaterales de los antiepilépticos.

- Considerar la eficacia del tratamiento: desaparición de las anomalías electroencefalográficas intercríticas y no la supresión de las crisis (con funcionabilidad normal) (Bender del Busto \& Hernández Toledo, 2017).

\section{Tratamiento en pacientes de edad avan- zada}

- Considerar tratamiento específico de etiología.

- Monitorizar función hepática y renal.

- Evaluar deglución efectiva.

- Inicio con dosis baja, titulación lenta.

- Evaluar posibles interacciones con otros fármacos.

- Intentar retiro de fármacos que puedan disminuir umbral convulsivante.

- Preferir monoterapia.

- Monitorización frecuente de reacciones adversas (Iragiien \& Simon, s.f).

En el tratamiento de pacientes en edad avanzada hay que tener consideraciones, ya que la función renal se deteriore con la edad progresivamente, el cerebro se vuelve sensible al efecto farmacológico de los anticonvulsivantes por perdida de los mecanismos homeostáticos del sistema nervioso central. Otros aspectos a considerar es el grado de deterioro cognitivo.

\section{Conclusiones}

La epilepsia es una condición importante, compleja por el tipo de fisiopatología, por sus diferentes conceptos, esta entre las 5 causas mas frecuentes por la que una persona acude a urgencias. Mientras más temprano se inicie la terapia antiepiléptica, mejor será el pronóstico, ya que lo tardío para su tratamiento supone una resistencia farmacológica. Es de suma importancia el inicio del tratamiento, ya que estudios han demostrado disminución en la resistencia y respuesta de los receptores NMDA a los medicamentos en etapas tardías.

Hay muchos medicamentos disponibles para tratar la epilepsia, sin embargo, su uso dependerá del grado de la misma y otras patologías que pueda presentar el paciente. El tratamiento para pacientes en edad avanzada, es mas complejo, ya que la persona a mayor edad va perdiendo ciertas condiciones neurológicas y fisiológicas, como el deterioro de la función renal, deterioro del grado cognitivo, entre otros.

La incidencia de tumores cerebrales en personas con epilepsia es del 4\%. La epilepsia es la primera manifestación clínica de los tumores cerebrales, entre el 30 y el $50 \%$ de los casos. Para estos casos se puede utilizar la farmacoterapia y el tratamiento quirúrgico el cual su efectividad esta entre un 40 y 90\% de liberación de crisis a los pacientes, pero esta última es más costosa.

\section{Bibliografía}

Bender del Busto, J. E., \& Hernández Toledo, L. (2017). Consideraciones en el tratamiento del paciente con epilepsia. Artículo de revisión. Revista Habanera de Ciencias Médicas, 16(6), 912-926.

González, W., \& Rodríguez, J. (2011). Manejo médico y farmacológico del estatus epiléptico. Acta neurol colomb, 11, 39-46.

Herrera, E. J., Suárez, J., Nieto, F., Surur, A., Pueyrredón, F., Palacios, C., \& Viano, J. (s.f). Meningiomas y epilepsia. Experiencia de 32 años.

Iragiien, P. L., \& Simon, E. (s.f). Epilepsia en adultos de edad avanzada: un desafío para el diagnóstico y el tratamiento.

LAPO ASENCIO, F. R. (2019). LA EPILEPSIA, SUS FACTORES DE RIESGO, MÉTODOS DE DIAGNÓSTICO, TRATAMIENTO INTEGRAL Y PRONÓSTICO. Machala.

Lozano Tangua, C. (2013). Patología dual en epilepsia del lóbulo temporal. 
Peña, S. (2018). Epilepsia focal asociada a tumor cerebral. Alerta, Revista científica del Instituto Nacional de Salud, 1(2), 87-90.

Víquez, L. E., \& Segura, J. (2017). Epilepsia del lóbulo temporal. Neuroeje, 30.
Volcy-Gómez, M. (2014). Epilepsia del lóbulo temporal mesial: fisiopatología, características clínicas, tratamiento y pronóstico. Rev Neurol, 38(7), 663667.

\section{CitAR ESTE ARTICULO:}

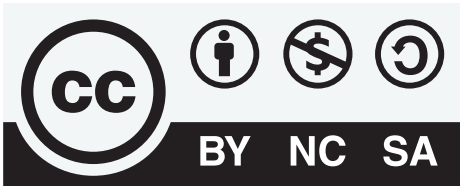

Morales Sánchez, A. F., Morales Sánchez, J. L., Guerra Hurtado, S. G., \& Navarrete Bolaños, K. A. (2021). Manejo farmacológico en epilepsia de origen tumoral. RECIMUNDO, 5(2), 80-88. https://doi.org/10.26820/recimundo/5.(2). abril.2021.80-88 